\title{
ELVIS: A Correlated Light-Field and Digital Holographic Microscope for Field and Laboratory Investigations
}

\author{
Taewoo Kim, ${ }^{3}$ Eugene Serabyn, ${ }^{1}$ Maximilian Schadegg, ${ }^{2}$ Kurt Liewer, ${ }^{1}$ Nathan Oborny, ${ }^{1}$ \\ J. Kent Wallace, ${ }^{1}$ Stephanie Rider, ${ }^{3}$ Manuel Bedrossian, ${ }^{3}$ Christian Lindensmith, ${ }^{1}$ and Jay Nadeau ${ }^{2 *}$ \\ ${ }^{1}$ Jet Propulsion Laboratory, California Institute of Technology, 4800 Oak Grove Dr., Pasadena, CA 91109 \\ ${ }^{2}$ Department of Physics, Portland State University, 1719 SW 10 ${ }^{\text {th }}$ Ave., Portland, OR 97201 \\ ${ }^{3}$ Andrew and Peggy Cherng Department of Medical Engineering, California Institute of Technology, 1200 E. California Blvd., \\ Pasadena, CA 91125 \\ *nadeau@pdx.edu
}

\begin{abstract}
This is the first of two articles on the Extant Life Volumetric Imaging System (ELVIS) describing a combined digital holographic microscope (DHM) and a fluorescence light-field microscope (FLFM). The instrument is modular and robust enough for field use. Each mode uses its own illumination source and camera, but both microscopes share a common objective lens and sample viewing chamber. This allows correlative volumetric imaging in amplitude, quantitative phase, and fluorescence modes. A detailed schematic and parts list is presented, as well as links to open-source software packages for data acquisition and analysis that permits interested researchers to duplicate the design. Instrument performance is quantified using test targets and beads. In the second article on ELVIS, to be published in the next issue of Microscopy Today, analysis of data from field tests and images of microorganisms will be presented.
\end{abstract}

Keywords: holographic microscopy, light-field microscopy, volumetric imaging, lenslet arrays, reconstruction software

\section{Introduction}

Recent technological developments, such as the FlowCytobot [1], have revolutionized the in situ study of microorganisms such as diatoms, plankton, and microalgae at the $10 \mu \mathrm{m}$ scale and above. However, smaller micron-scale organisms (bacteria and archaea) remain a neglected group of organisms for which limited environmental in situ imaging has been performed [2], largely because of the challenges placed on the imaging system.

Prokaryotic life is limited by nutrient diffusion into the cell, restricting most bacteria and archaea to sizes in the range of $0.4-2.0 \mu \mathrm{m}$ [3]. Not only are cells small, but they lack distinctive features such as nuclei and other membrane-bound organelles, making them difficult to distinguish microscopically from debris (Figure 1). Imaging systems with sufficient resolution to identify objects at this size scale generally have very limited field of view and depth of field. This makes it difficult to observe a large number of interactive microorganisms and requires active focus and stage motion to track a single organism for even short times. Because of the need for instantaneous volume imaging, implementation of such features in a system for field use is daunting even if equipped with adaptive lenses.

In this paper, we report a multi-modal microscope called the Extant Life Volumetric Imaging System (ELVIS) designed for field use that integrates two modalities into a common instrument: digital holography microscopy (DHM) and fluorescence light-field microscopy (FLFM). ELVIS provides synchronous volumetric imaging of three types: fluorescence, intensity (bright-field), and quantitative phase. Both the DHM and FLFM components of the combined instrument are closely based on previously published designs $[4,5]$.

DHM is an interferometric technique that results in full electric field measurements of the sample volume. Offaxis holographic images may be reconstructed into intensity (bright-field) and quantitative phase images plane-by-plane through volumes $\sim 100$ times deeper than those imaged using ordinary bright-field microscopy [6]. Amplitude and brightfield images are equivalent. However, quantitative phase imaging is an emerging technique in biology and materials science and has no direct counterpart in ordinary light microscopy. In quantitative phase imaging the phase shift of the light wave passing through the specimen is proportional to the product of the specimen's thickness and the difference in the refractive index from the surrounding medium. The phase shift information can be used to differentiate bacteria from minerals, to identify malignant cells in a tissue sample, to monitor the flux of water into and out of cells via ion channels, and to monitor neuronal function $[7,8]$.

Fluorescence microscopy is a ubiquitous tool in biology that allows for specific labeling of subcellular structures, enzymatic processes, individual proteins or nucleic acid sequences, and much more. A combined intensity/quantitative phase/ fluorescence microscope would offer one-of-a-kind volumetric analysis. The problem lies in adding fluorescence capability to a technique such as DHM that is focus-free. Because DHM works on the principle of interfering coherent light, it does not directly support volumetric fluorescence imaging, and when coupled with fluorescence using traditional microscopes, imaging has been limited to a single plane $[9,10]$. This means that either most of the sample is missed by the fluorescence analysis or that it must be filtered onto a flat substrate, which disturbs correspondence between the DHM and fluorescence images.

In FLFM, the 3D light field of the sample is transformed into a $2 \mathrm{D}$ image using a microlens array, thus enabling a $2 \mathrm{D}$ camera to image the 3D sample volume [11-13]. Computational reconstruction is then used to generate the $3 \mathrm{D}$ image of the sample from its 2D light-field image. FLFM suffers the tradeoff of reduced lateral resolution compared to conventional microscopy carried out with similar numerical apertures. 


\section{First $\gg>>$}

\section{HIGH THROUGHPUT SEM System}

\section{Navigator-10T}

(6) 10 times faster than
conventional FE SEMs

Direct electron detection technology and Dual channel imaging (SE\&BSE)

Intelligent atlas and cross-scale material characterization

(1) Big data intelligent analysis and Al computing
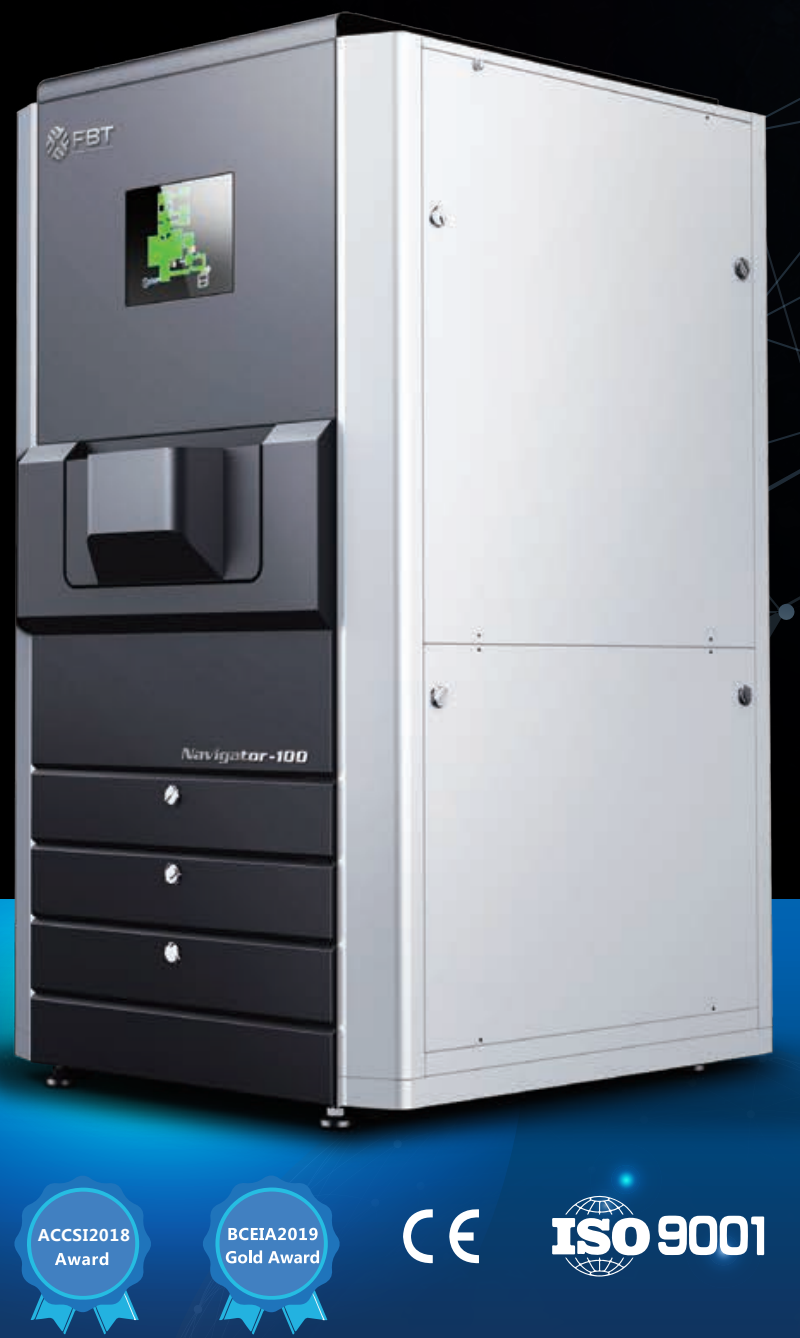

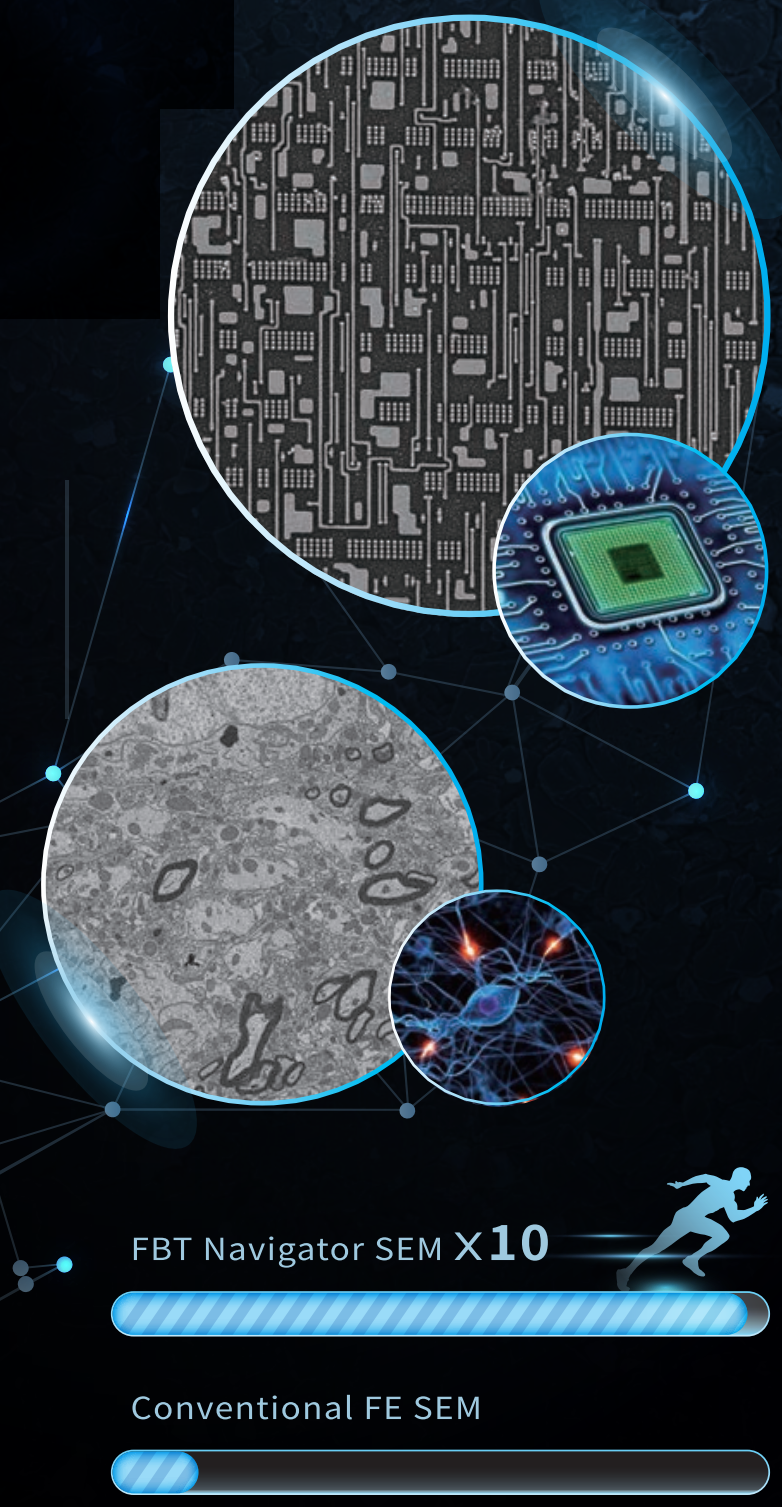

CHINA FOCUS EBEAM TECHNOLOGY(BEIJING) CO. ,LTD Tel: +86010 67832505 ext 813

\section{SINGAPORE}

FOCUS EBEAM TECHNOLOGY PTE.LTD Tel: +65 63498868 ext 8921 
(a)

\section{Eukaryotes}

Membraneenclosed nucleus
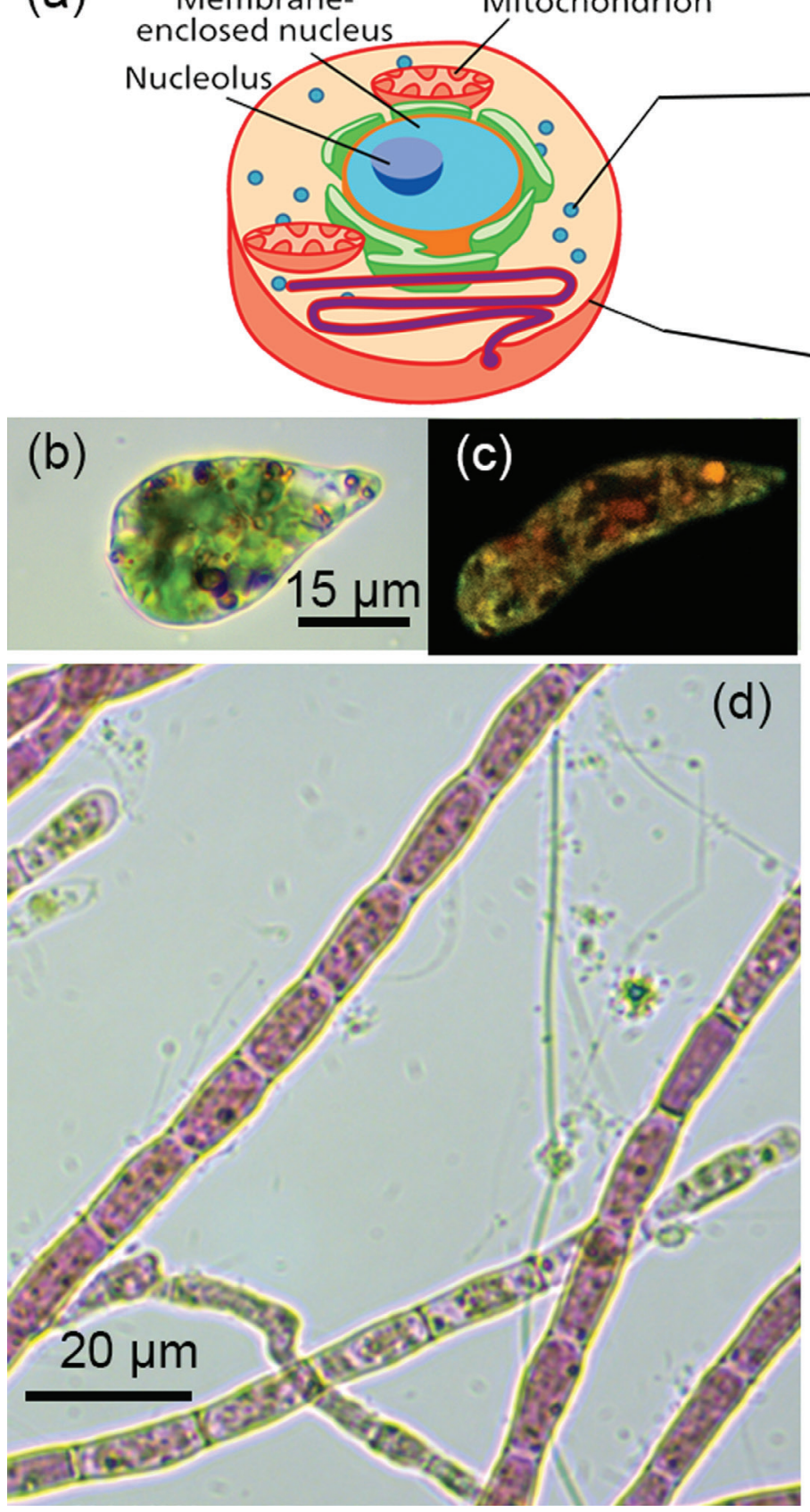

(e)

\section{Prokaryotes}

\section{Nucleoid}

Ribosomes

Capsule (some prokaryotes)
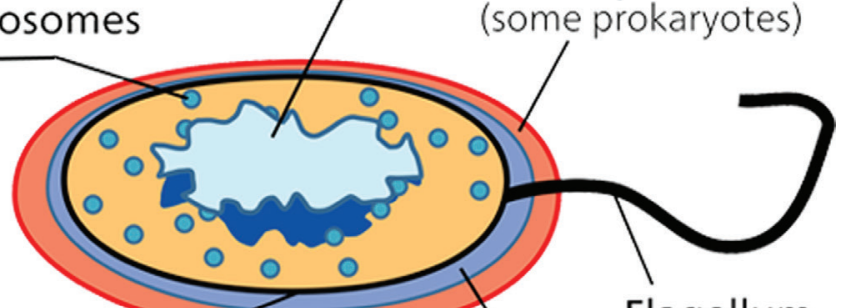

Flagellum

\section{Cell Wall}
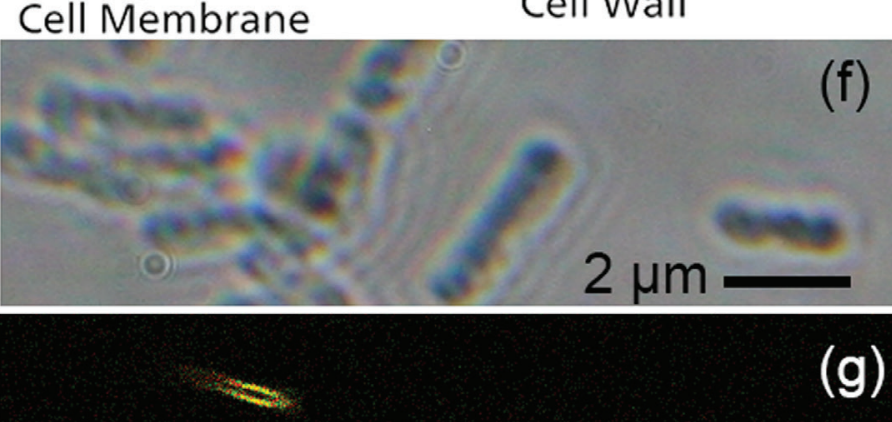

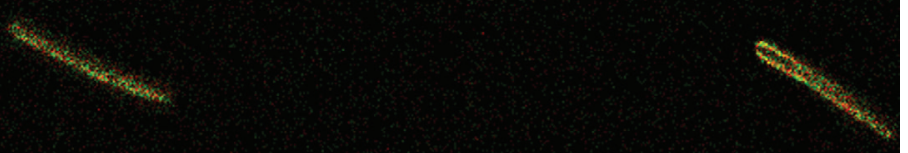

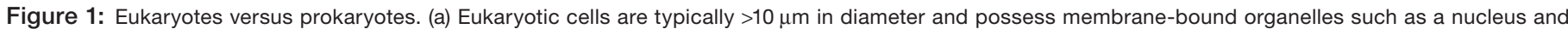

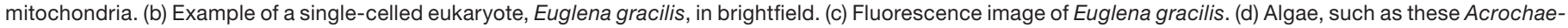

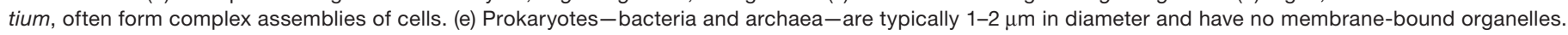

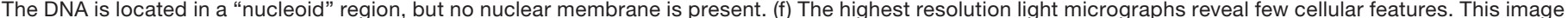

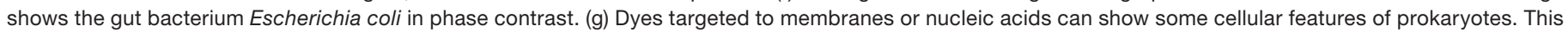
image shows the soil bacterium Bacillus subtilis labeled with the membrane dye FM1-43.

Depending on instrument goals, this can be partially mitigated by increasing the detector array size and appropriate lenslet array selection. However, the capability of synchronous volumetric imaging could offer game-changing prospects for many applications, particularly when combined with a DHM that provides high resolution in both amplitude and phase imaging.
Here we present a design for a combined DHM/FLFM instrument and quantify its performance using test samples. The trade-offs in volume versus resolution are discussed and future improvements suggested. In a second paper to be published in Microscopy Today, we will present data collected from cultured bacterial and in situ environmental microorganisms 
Table 1: Optical components for ELVIS. The numbered parts are shown in the schematic in Figure 2.

\begin{tabular}{|c|c|c|c|c|c|c|c|}
\hline & Illumination & Collimator & Filters & Beamsplitter & Objectives & Tube lens & Camera \\
\hline DHM & $\begin{array}{l}405 \mathrm{~nm} \\
\text { diode laser } \\
\text { (Thorlabs } \\
\text { S1FC405) } \\
\text { (1a) }\end{array}$ & $\begin{array}{l}100 \mathrm{~mm} \text { focal } \\
\text { length } \\
\text { achromat; } 1 " \\
\text { diameter } \\
\text { Newport } \\
\text { Optics } \\
\text { PAC052AR.13 } \\
\text { (2a) }\end{array}$ & $\begin{array}{l}405 \times 10 \mathrm{~nm} \\
\text { bandpass } \\
\text { filter (3) }\end{array}$ & $\begin{array}{l}\text { BS1: } 425 \mathrm{~nm} \\
\text { shortpass } \\
\text { dichroic } \\
\text { beamsplitter } \\
25 \times 36 \mathrm{~mm} \\
\text { (Thorlabs } \\
\text { DMSP425R) }\end{array}$ & $\begin{array}{l}\text { Pair (science } \\
\text { beam/ } \\
\text { reference } \\
\text { beam): } 10 \mathrm{~mm} \\
\text { focal length } \\
\text { achromats; } \\
6.25 \mathrm{~mm} \\
\text { diameter } \\
\text { (masked to } \\
5 \text { mm) } \\
\text { (Edmund } \\
\text { Optics 47689) }\end{array}$ & $\begin{array}{l}150 \text { mm focal length } \\
\text { achromat; 2" } \\
\text { diameter } \\
\text { (Newport Optics } \\
\text { PAC086AR.13) (7a) }\end{array}$ & $\begin{array}{l}2464 \times 2056 \\
\text { pixels } \\
\text { (windowed to } \\
2048 \times 2048 \text { in } \\
\text { operation) } \\
3.45 \times 3.45 \mu \mathrm{m} \\
\text { pixels } \\
\text { (AlliedVision } \\
\text { GT2460) (10a) }\end{array}$ \\
\hline FLFM & $\begin{array}{l}470 \text { nm LED } \\
\text { (Thorlabs } \\
\text { M470L3) } \\
\text { (1b) }\end{array}$ & $\begin{array}{l}20.1 \mathrm{~mm} \text { focal } \\
\text { length; } 1 " \\
\text { diameter } \\
\text { (Thorlabs } \\
\text { ACL2520U- } \\
\text { A)(2b) }\end{array}$ & $\begin{array}{l}\text { Excitation: } \\
469 \pm 35 \mathrm{~nm} \\
\text { bandpass } \\
\text { (Thorlabs } \\
\text { MF469-35) } \\
\text { (4) } \\
\text { Emission: } \\
500 \mathrm{~nm} \\
\text { longpass } \\
\text { (Thorlabs } \\
\text { FELH0500) } \\
\text { (5) }\end{array}$ & $\begin{array}{l}\text { BS2: } 490 \mathrm{~nm} \\
\text { shortpass } \\
\text { dichroic; } \\
25 \times 36 \mathrm{~mm} \\
\text { (Thorlabs } \\
\text { DMSP490/R }\end{array}$ & $\begin{array}{l}\text { Same as } \\
\text { DHM;only use } \\
\text { one of the } \\
\text { objectives } \\
\text { (6) }\end{array}$ & $\begin{array}{l}200 \text { mm focal length } \\
\text { achromat; 2" } \\
\text { diameter } \\
\text { (Thorlabs AC508- } \\
\text { 200-A) (7b) } \\
\text { Lenslet array } \\
3.75 \text { mm focal } \\
\text { length and } 125 \mu \mathrm{m} \\
\text { pitch (RPC } \\
\text { Photonics } \\
\text { RPC125-f30) (8) } \\
\text { 2:1 Telecentric } \\
\text { optical relay (Opto } \\
\text { Engineering } \\
\text { TC23-016) (9) }\end{array}$ & $\begin{array}{l}\text { Same as DHM } \\
\text { or RGB } \\
\text { camera of } \\
\text { choice (10b) }\end{array}$ \\
\hline
\end{tabular}

to demonstrate practical applications of the combined DHM/ FLFM system.

\section{Materials and Methods}

The design of the DHM side of the instrument has been described in detail [4], with small differences in component selection for the combined DHM/FLFM instrument (Table 1). The DHM/FLFM system was developed with the goal of using a single objective, or a set of objectives, for both modes of the instrument, but with otherwise independent optics. In principle the DHM light source can be used for the FLFM illumination, but in practice there are advantages, described below, to using separate illumination capability for both modes. Note that one of our goals was to make use of relatively simple and inexpensive objective lenses to reduce the cost, complexity, and number of optical surfaces involved. Figure 2 shows a schematic and photo of the instrument, illustrating the instrument elements listed in Table 1.

\section{Results}

ELVIS standardized sample testing. Amplitude resolution laterally $(x, y)$ and axially $(z)$, depth of field, and phase sensitivity were all measured using U.S. Air Force (USAF) test targets [Edmund Optics SKU 58-198]. DHM-mode amplitude reconstructions without noise subtraction showed lateral resolution better than $0.9 \mu \mathrm{m}$ (Figure 3a). No measurable loss of resolution in the reconstructed images was seen at a range of $900 \mu \mathrm{m}$ in $z$. Since this exceeded the depth of our sample chambers, we did not measure depth of field farther. A measure of axial resolution was obtained by translating the micrometer stage a known distance in $z$ and comparing this known distance with the position of best reconstructed focus. These values should differ by the square of the magnification of the system (approximately 218). A best fit to these values gives a slope of 228 with fit residuals of $9.6 \mu \mathrm{m}$ at the sample, which corresponds to $4.4 \mu \mathrm{m}$ axial resolution (Figure $3 \mathrm{~b}$ ).

A phase target consisting of patterns of known thickness was imaged, and the difference between the averages of a patch within the largest square and a similar patch just outside was measured (Figure 3c). The graph in Figure $3 \mathrm{~d}$ plots the measured phase delay in $\mathrm{nm}$ versus the actual thickness in $\mathrm{nm}$. The best fit slope is $0.52+/-0.02$ (with a small offset). The slope corresponds to $n-1$, where $n$ is the index of refraction of the phase material, corresponding to the manufacturer's value. The residuals are $4 \mathrm{~nm}$, or $1 \%$ of a wave.

On the FLFM side, the field of view was measured to be $790 \times 660 \mu \mathrm{m}$, which exceeded that of the DHM. The two fields of view overlapped to a large extent, but not completely. Figures $3 e$ and $3 f$ show raw and reconstructed USAF target images obtained with the FLFM that resolved line group 6,5, implying a resolution after reconstruction of $4.9 \mu \mathrm{m}$. The lateral resolution degraded with axial offset, leading to a depth of field of $150 \mu \mathrm{m}$ with a resolution of $5.5 \mu \mathrm{m}$, and $300 \mu \mathrm{m}$ for $7.0 \mu \mathrm{m}$ resolution.

Axial resolution was also measured using $100 \mathrm{~nm} \mathrm{SiO}_{2}$ beads (Polysciences, Inc. 24041-10). Amplitude and phase 


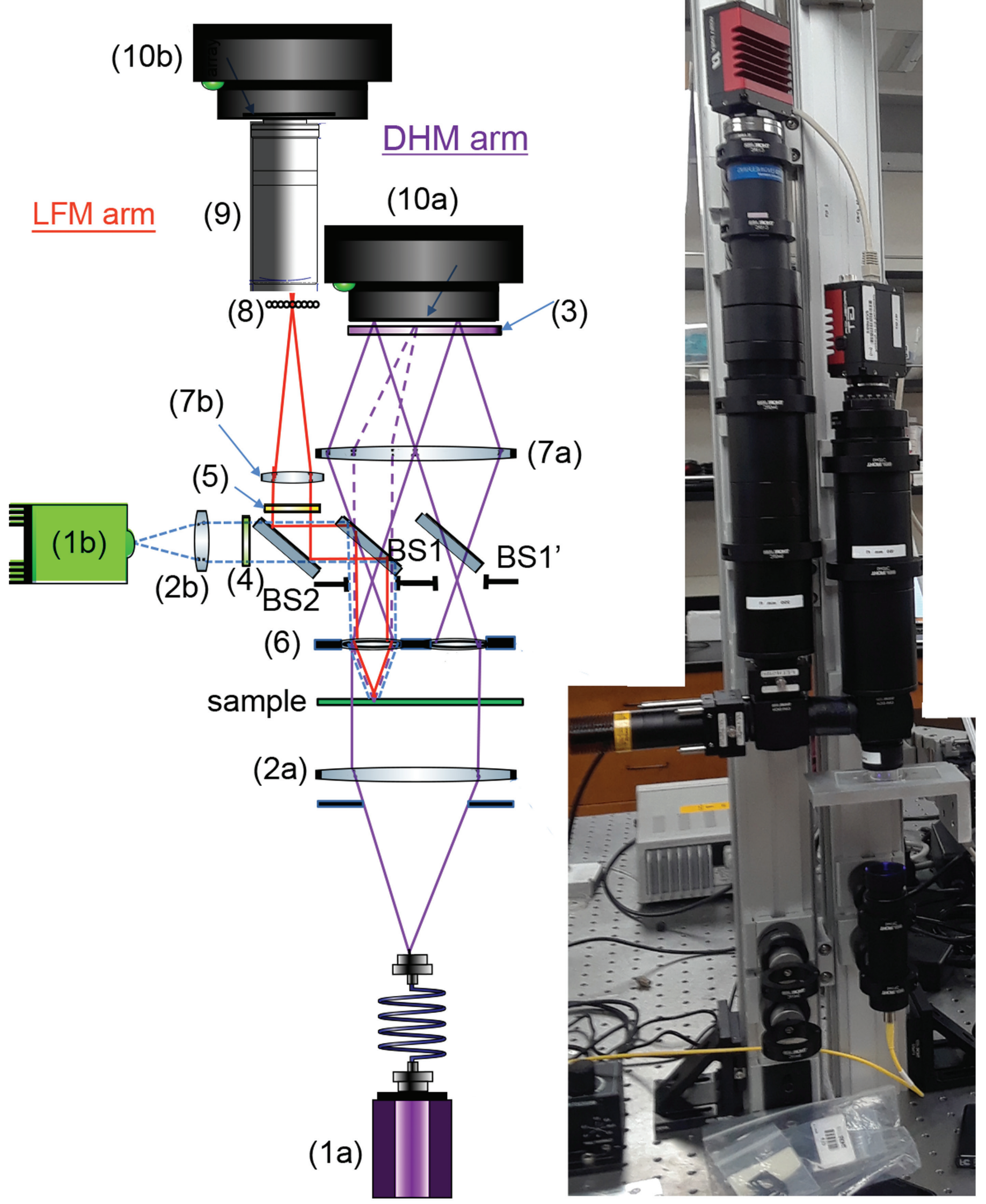

Figure 2: Schematic (left) and photo (right) of the instrument, with labels corresponding to the parts in Table 1. Note: BS1 and BS1' are the same optic. 
(a)

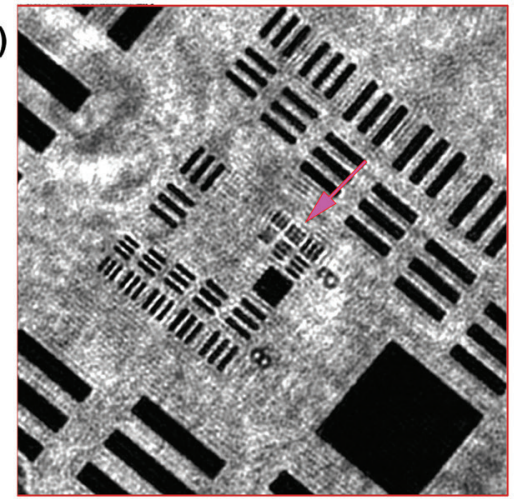

(c)

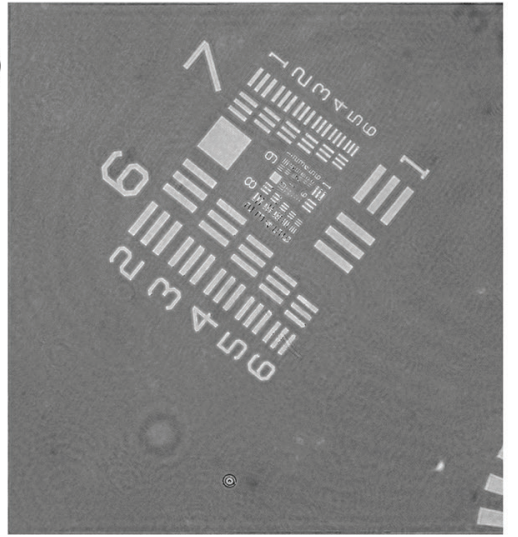

(e)

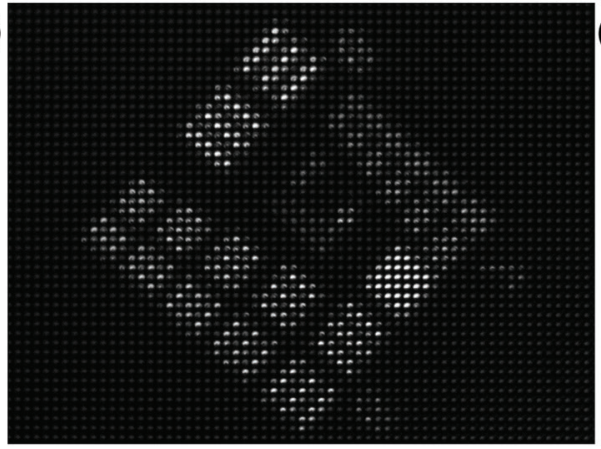

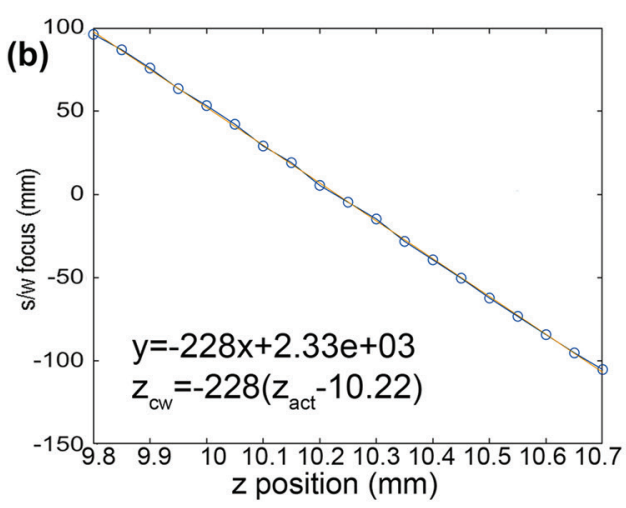
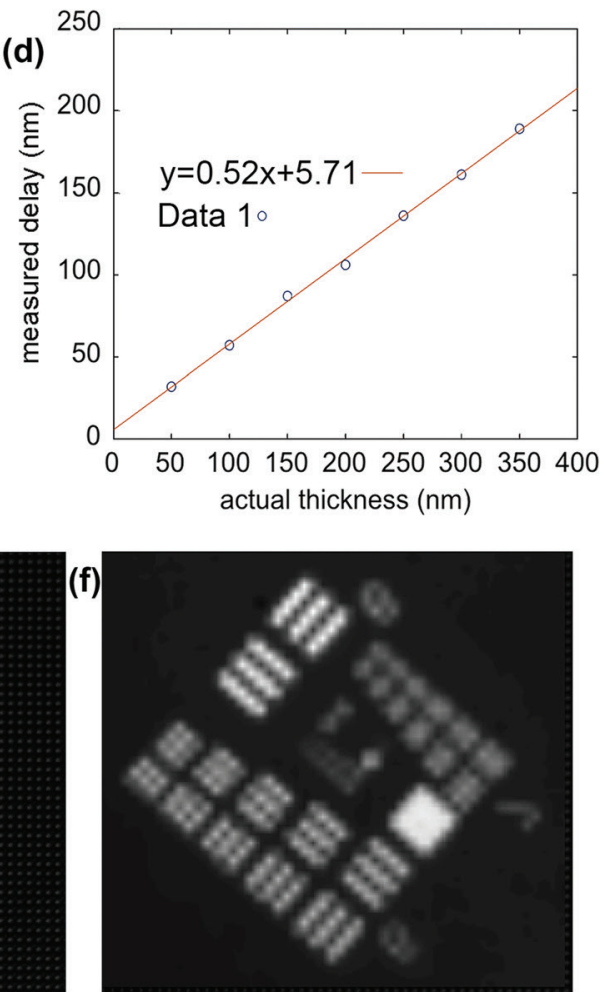

Figure 3: DHM/FLFM test target (TT) images. Only a portion of the field of view is shown. The total field of view was $478 \times 478 \mu \mathrm{m}$. (a) Amplitude reconstruction of USAF TT showing resolution better than group 9, element $2(0.87 \mu \mathrm{m}$ line widths). (b) The reconstructed best-focus position versus the actual position, allowing an estimate of axial resolution of $4.4 \mu \mathrm{m}$ (see text). (c) Phase reconstruction of USAF phase TT with known widths. (d) Phase delay in $\mathrm{nm}$ versus the actual thickness in $\mathrm{nm}$ (data points shown as open circles) with best linear fit, permitting estimate of phase resolution (see text). (e) Raw FLFM image of USAF TT. (f) Reconstructed FLFM image showing resolution of group 6, element 5 (4.92 $\mu \mathrm{m}$ line widths).

resulting low sensitivity so that even labeled bacteria could not be identified under the FLFM; and (b) monochromatic fluorescence that did not permit distinguishing dye labeling from chlorophyll autofluorescence, or the use of 2 different dyes. Simple modifications resulted in improved performance. The 3D-printed objective lens holder was found to show significant autofluorescence, so it was replaced by a holder made of anodized aluminum. The use of an RGB camera was also used on the FLFM side to distinguish chlorophyll from common dyes (Figure 5) without the need to insert or change filters.

Software. Acquisition and real-time DHM reconstruction were performed using a custom package, DHMx, written by our group. DHMx runs under Linux and is open-source (https://github.com/ dhm-org/dhm_suite). FLFM reconstruction was performed using another group's open-source package [14]. Full amplitude and phase reconstructions were made using custom Fiji plug-ins that we have published previously [15].

\section{Discussion}

The combined DHM/FLFM system provides multimodal volumetric imaging, but trade-offs were made to permit the combination, which can be further optimized for particular applications.

The first major trade-off in the design of the instrument was the choice to use a shared set of objectives for both modes of the microscope. In early development we considered both this shared-objective design and a second design that used two fully independent microscopes observing the same volume at crossed angles. The shared objectives architecture ensures straightforward

reconstructions of the beads are shown in Figures $4 a$ and $4 \mathrm{~b}$. Both images were de-noised by translating the stage while imaging and using the median image to subtract amplitude background; a reference hologram containing no beads was used to remove noise in phase. These procedures are described in detail elsewhere [15]. Amplitude and phase $x-z$ slices are shown in Figures $4 \mathrm{c}$ and $4 \mathrm{~d}$.

Fluorescence sensitivity was ascertained using fluorescent $\mathrm{SiO}_{2}$ beads (Polysciences, Inc. 24330-15). Figure 4e shows a raw image of $3 \mu \mathrm{m}$ beads, and Figure $4 \mathrm{f}$ shows a maximum intensity projection through the sample.

Early issues with the instrument that were identified and ameliorated were (a) high background fluorescence, with co-registration of the fields of view. The choice of objectives then sets both the capability and to some extent the size of the instrument. We have been working with simple aspheric and achromat objectives in order to make an instrument that is more rugged for field use and to avoid optical elements that might adversely affect the ability to obtain high fringe contrast in the DHM. Simple aspheric objectives are sufficient for the DHM, but their chromatic aberration can move the focus for many desired wavelengths far enough out of focus as to be effectively useless for imaging broad bands of light. Simple achromatic doublet objectives help mitigate this, and compound-apochromat or super-apochromat objectives could improve this further. Compound objectives must be selected 

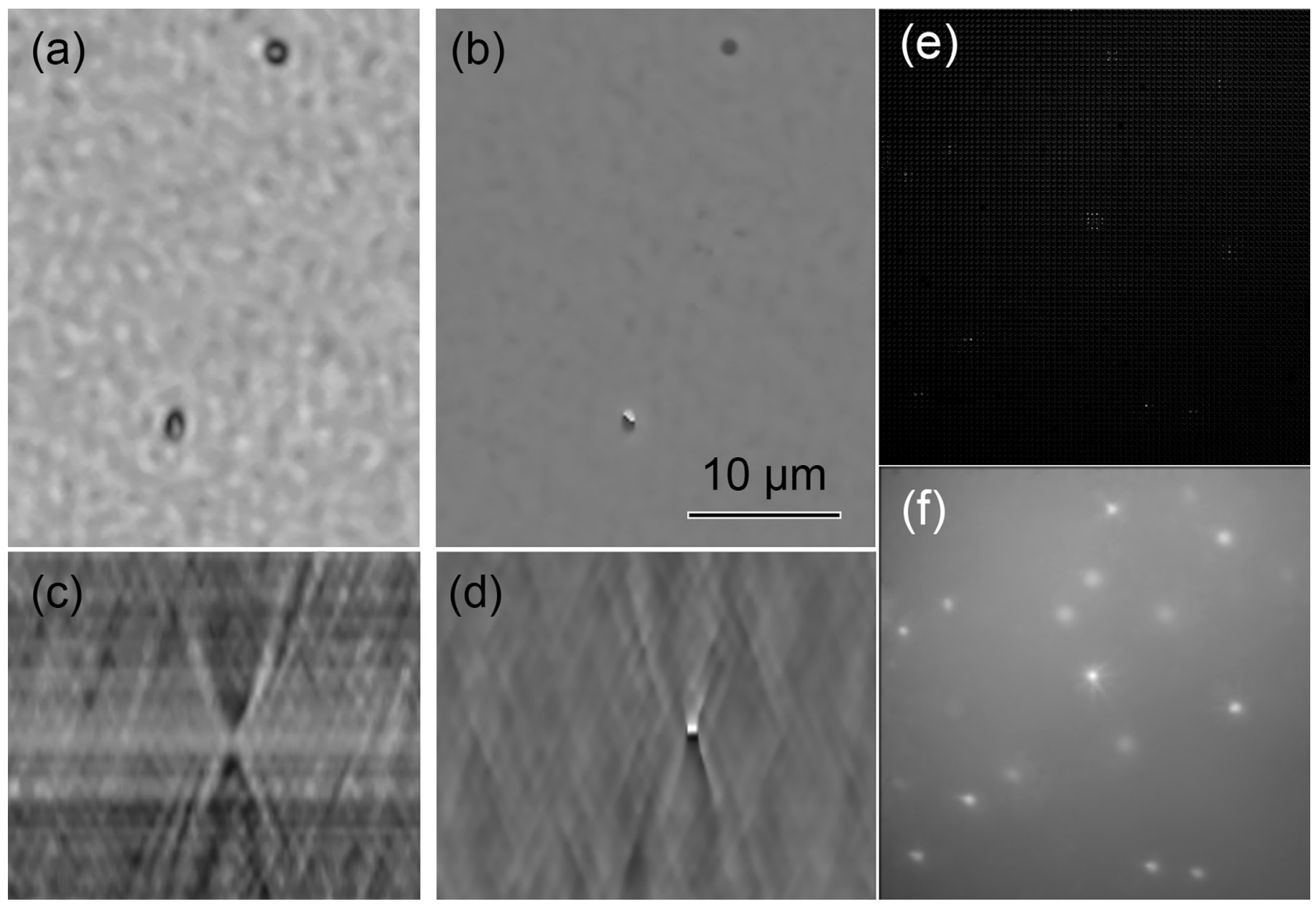

Figure 4: Resolution and fluorescence sensitivity using beads. (a) $100 \mathrm{~nm}$ beads in a single plane amplitude reconstruction. (b) $100 \mathrm{~nm}$ beads in phase. (c) Amplitude in the xz plane of a single bead. (d) Note how the phase flips from dark to light across the focal plane; this is a manifestation of the Gouy phase anomaly. (e) Raw FLFM image of $3 \mu \mathrm{m}$ fluorescent beads. (f) Maximum intensity projection of reconstructed LFM bead image.

carefully-many that are optimized for bright-field microscopy have elements that affect polarization and thus the interference in the DHM. We have found that at least some high-quality objectives designed for metallurgy provide both good DHM and fluorescence performance.

A second significant trade-off is in the lateral resolution of the fluorescence microscope mode. In the implementation shown here the same model of camera was used for both microscopes for low cost and because our intended long-term application as an in situ planetary instrument drastically limits the rate and volume of data return compared to a laboratory environment [16]. Increasing the pixel count and enlarging the sensor size on the FLFM detector is a straightforward way to improve the lateral resolution without a loss of field of view. The FLFM camera and detector could even be chosen to provide resolution, depth of field, and field of view comparable to the DHM, at the expense of a substantially increased data volume and likely a small increase in power consumption.

One might ask then why the dual instrument is still valuable at the design point where both have comparable resolution and field. Why not just the FLFM? The DHM mode offers labelfree imaging of many otherwise transparent or translucent objects in the field, providing a survey capability that does not require any a priori knowledge of the characteristics of the objects in the field. Fluorescence microscopy typically requires some knowledge of the objects of interest-either excitation wavelengths of autofluorescent elements or chemical characteristics so that appropriate dyes can be selected. The DHM mode can readily detect objects that have only a few percent index difference to the surrounding medium and provides diffraction-limited resolution of the volume.

Another trade-off in the design of the instrument is the selection of a color versus monochrome camera on the FLFM side. The initial instrument described here was optimized for broad detection and discrimination of cells from mineral objects and so was designed around a small group of dyes. For fully correlated real-time observation of all dyes simultaneously, a color camera filter set with isolated excitation and detection bands is valuable, but some applications could use a monochrome camera and selectable single-band filters.

\section{Conclusion}

The combined DHM-FLFM allows for fluorescence detection in addition to volumetric amplitude and phase imaging. 
(a)

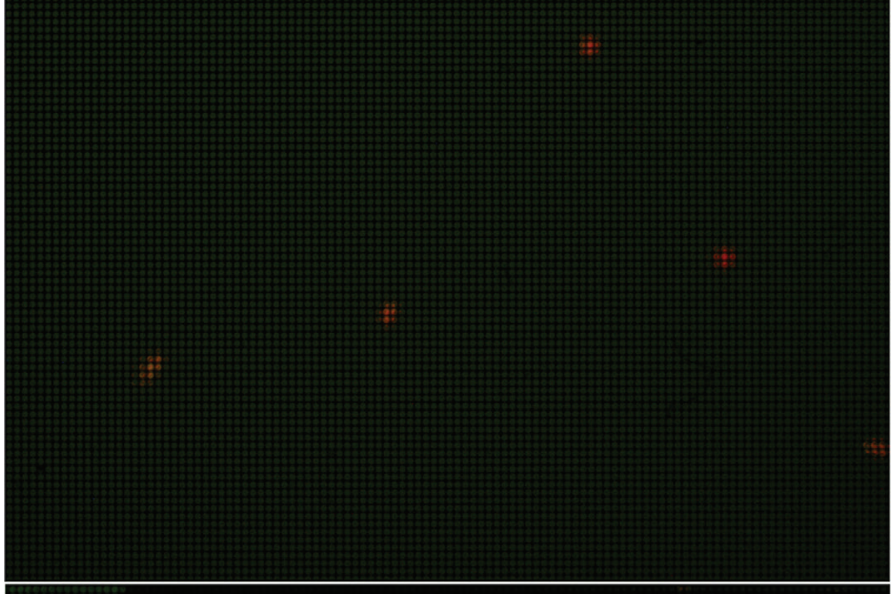

(c)

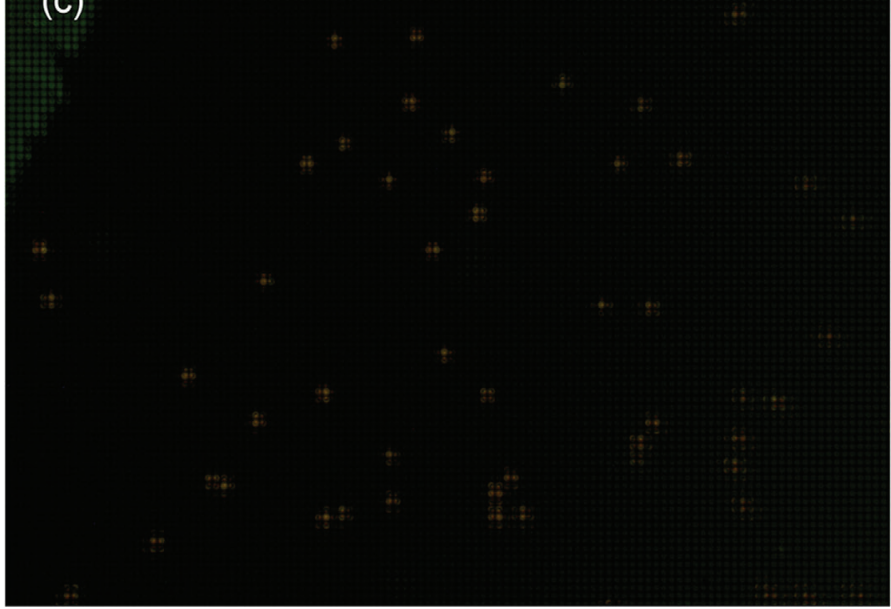

(b)

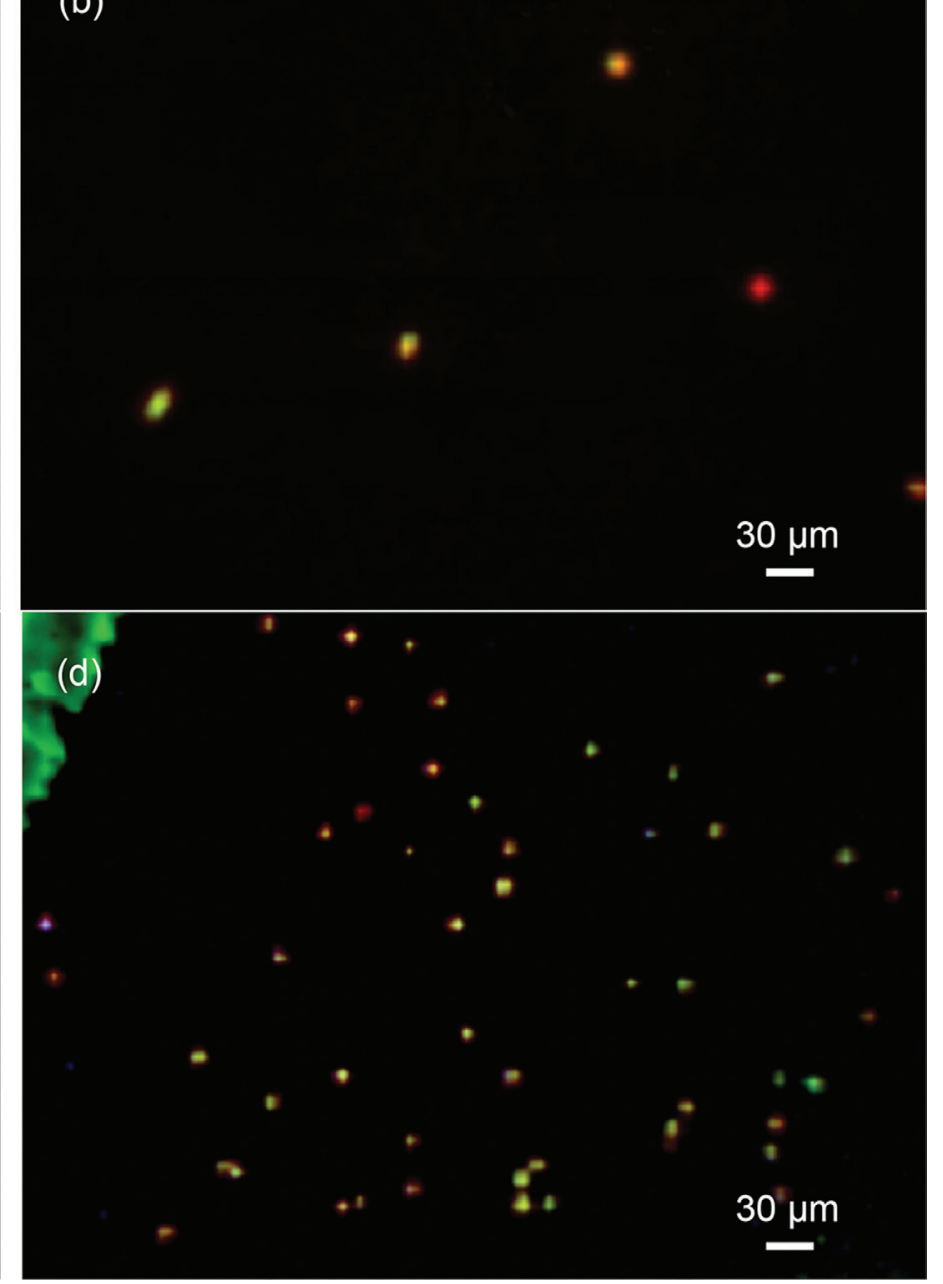

Figure 5: Use of an RGB camera with long-pass emission to discriminate acridine orange (AO) from chlorophyll. The samples are Euglena gracilis raw (a) and reconstructed (b), and Chlamydomonas sp. raw (c) and reconstructed (d).

Although the spatial resolution of the FLFM is lower, the fluorescent signal can be matched to the higher-resolution DHM images to confirm cellular localization. The addition of RGB fluorescence detection and pulsed illumination will greatly improve the fluorescence capability by adding specificity and reducing phototoxicity and photobleaching. The simple design and open-source software make this instrument readily accessible to potential users.

\section{Acknowledgements}

Portions of this work were performed under a contract from the Jet Propulsion Laboratory, California Institute of Technology and at the Jet Propulsion Laboratory, California Institute of Technology under contract with the National Aeronautics and Space Administration. Portions of this work were supported by NSF Award \#1828793.

\section{References}

[1] RJ Olson and HM Sosik, Limnol Oceanogr 5 (2007) 195-203.

[2] I Dickinson et al., in Their World: A Diversity of Microbial Environments, Vol. 1, ed. CJ Hurst, Springer, 2016, 341-81.
[3] WF Marshall et al., BMC Biol 10 (2012) 101-23.

[4] JK Wallace et al., Optics Express 23 (2015) 17367-78.

[5] G Serabyn et al., in 2019 IEEE Aerospace Conference 10.1109/AERO.2019.8741627 (2019) 1-7.

[6] E Cuche et al., Interferometry in Speckle Light (2000) 213-18.

[7] P Marquet et al., Neurophotonics 1 (2014) 020901.

[8] K Lee et al., Sensors 13 (2013) 4170-91.

[9] N Pavillon et al., J Biophoton 3 (2010) 432-36.

[10] X Quan et al., Opt Rev 22 (2015) 349-53.

[11] M Broxton et al., Optics Express 21 (2013) 25418-39.

[12] R Prevedel et al., Nature Meth 11 (2014) 727-30.

[13] E Serabyn, Appl Optics 58 (2019) A273-82.

[14] J Bolan et al., SoftwareX 5 (2016) 101-06.

[15] D Cohoe et al., Front Physics 7 (2019) 94.

[16] KP Hand et al., Project Engineering Team Report of the Europa Lander Science Definition Team (2017) https:// europa.nasa.gov/resources/58/europa-lander-study-2016report. 